\title{
Simplistic calculation of the tunneling splittings for proton transfer in malonaldehyde and formic acid dimer using the one-dimensional Schrödinger equation
}

\author{
Denis S. Tikhonov ${ }^{a, b}$, \\ ${ }^{a}$ Deutsches Elektronen-Synchrotron DESY, Notkestr. 85, D-22607 Hamburg, Germany; \\ ${ }^{b}$ Institute of Physical Chemistry, Christian-Albrechts-Universität zu Kiel, Max-Eyth-Str. 1, D-24118 Kiel, Germany \\ ${ }^{c}$ Free Moscow University, https: // freemoscow. university/
}

June 7,2021

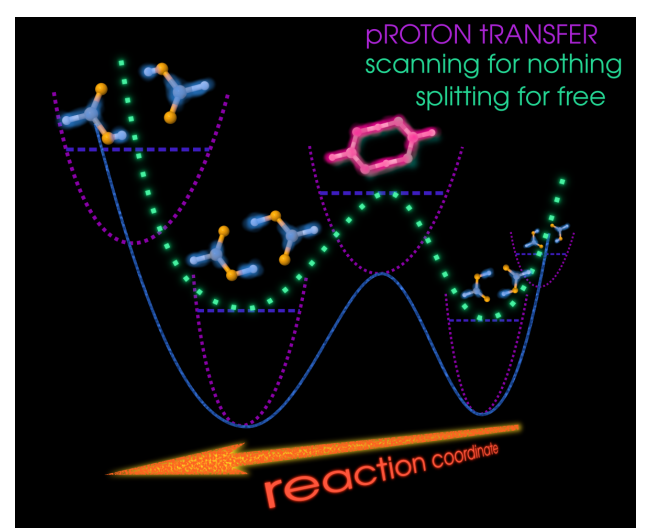

\begin{abstract}
In this manuscript we present an approach for computing tunneling splittings for large amplitude motions. The core of the approach is a solution of an effective one-dimensional Schrödinger equation with an effective mass and an effective potential energy surface composed of electronic and harmonic zero-point vibrational energies of small amplitude motions in the molecule. The method has been shown to work in cases of three model motions: nitrogen inversion in ammonia, single proton transfer in malonaldehyde, and double proton transfer in the formic acid dimer. In the current work we also investigate the performance of different DFT and post-Hartree-Fock methods for prediction of the proton transfer tunneling splittings, quality of the effective Schrödinger equation parameters upon the isotopic substitution, and possibility of a complete basis set (CBS) extrapolation for the resulting tunneling splittings.
\end{abstract}

\section{Introduction}

Large amplitude motions (LAM) are ubiquitous in most molecular systems, they are responsible for conformational interconversion and even for chemical reactions, such as in the cases of tautomerization. [1 5] One of the most prominent LAM manifestation is the tunneling splitting of the ground vibrational state, whenever a motion between equivalent minima is involved. Such splittings can be observed, for instance, using microwave (MW) or millimeter wave (MMW) rotational spectroscopy. [6] A knowledge of the LAM and of its characteristics can be helpful for the interpretation of the experimental data. [13, 16, 17, 15] 11

An important chemical reaction, which is also a LAM, is the proton transfer via the Grotthuss mechanism: 1, 3. 7 .

$$
\mathrm{R}-\mathrm{O}-\mathrm{H} \cdots \mathrm{O}-\mathrm{R}^{\prime} \leftrightharpoons \mathrm{R}-\mathrm{O} \cdots \mathrm{H}-\mathrm{O}-\mathrm{R}^{\prime}
$$

The canonical systems with such reactions are malonaldehyde (MA) and formic acid dimer (FAD) shown in Fig. 1 MA has a large tunneling splitting of $21 \mathrm{~cm}^{-1}$, which is well known from experiments for more than 20 years. 8 , 9 FAD is a system investigated theoretically for a long time, 18, 19, but the experimental tunneling splitting (approx. $0.01 \mathrm{~cm}^{-1}$ ) was obtained only recently. [20, 21, 10, 11]

Both these systems have been thoroughly investigated using various theoretical approaches. The low dimensional treatment of proton transfer and some of the coupled motion have been shown to give reasonable splitting estimates for MA, 22] while for FAD such applications were less successful. 23, 24. Applications of the reaction surface Hamiltonian (RSH) models 25, 27, and of anharmonic normal mode-based treatments 28, 31, 19, 32, have yielded in good results for both MA and FAD. As expected, the full-dimensional quantum Monte-Carlo simulations provide results of the 


\section{Malonaldehyde (MA)}

\section{Formic Acid Dimer (FAD)}
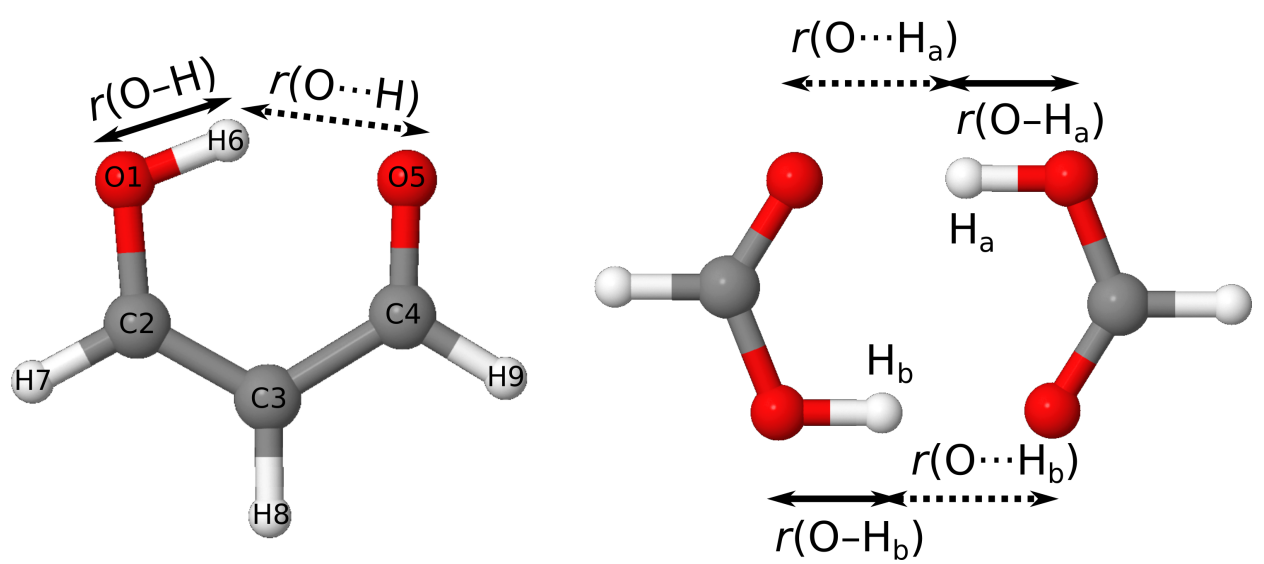

Figure 1: Structures and proton transfer coordinates of malonaldehyde (MA) and formic acid dimer (FAD). The atomic color schemes are the same for MA and FAD.

experimental quality for MA. 33, 34, 31] For MA and FAD, instanton approaches have been shown to produce accurate results. $35-39$

In this paper we present a modified version of another computational approach provided in Refs. [40, 41, 17. It assumes adiabatic separation of the LAM from the other vibrational modes, that we will further refer to as smallamplitude motions (SAM). The latter are to be treated in the harmonic approximation. Our procedure requires two types of calculations to be done: one-dimensional (1D) relaxed potential energy surface (PES) scans and vibrational frequency calculations in the harmonic approximation. Both computational procedures are known by users of quantumchemical packages, and thus the presented procedure can become an interesting one for members of the high-resolution molecular spectroscopy community. We apply the proposed approach to the nitrogen inversion in ammonia to prove the workability of the method, and then we use it to calculate tunneling splittings for the proton transfer motion in MA and FAD at various quantum-chemical approximations employing the def2-TZVPP basis set.

\section{Computational procedure}

\subsection{Quantum chemical calculations}

The quantum chemical calculations in the present work were done using Orca 4 software. 42 We have applied the following DFT functionals: PBE, 43. PBE0, 44] BLYP and B3LYP, 45, 47] CAM-B3LYP, 48, B2PLYP, 49] and mPW2PLYP. [50] From the post-Hartree-Fock methods, we have applied MP2, SCS-MP2, and all-electron CCSD(T) (ae-CCSD $(\mathrm{T})$ ). 51 53. Some of the calculations were done employing S. Grimme's D3 dispersion correction with Becke - Johnson (BJ) damping. 54 We have applied the resolution-of-identity (RI) technique in order to reduce the computational efforts: we have applied the RIJCOSX method for MA, FAD and B3LYP-D3BJ and CAM-B3LYP calculations of ammonia, while for MP2 and B2PLYP calculations of ammonia we have used the RI-JK method. 55 61 Basis sets of our choice were def2-nVP, def2-nVPD ( $\mathrm{n}=\mathrm{S}, \mathrm{TZ}, \mathrm{QZ})$, and def2-TZVPP, as well as their corresponding auxiliary basis sets. 62.65

\subsection{Theoretical framework}

The treatment of the LAM that we implemented resembles the procedure introduced in Refs. 40, 41. It is a mixture of the RSH approach, 66, 69, 3, 26, with the kinetic energy expression taken from the Meyer - Günthard Hamiltonian. [70] The Hamiltonian is expressed as

$$
\hat{H}=-\frac{1}{2} \hat{p}_{\xi} G(\xi) \hat{p}_{\xi}+V(\xi)+\hat{H}_{\mathrm{b}}(\xi)
$$

where $\xi$ is the LAM coordinate, $\hat{p}_{\xi}=-i \hbar \partial / \partial \xi=-i \hbar \partial_{\xi}$ is the momentum conjugated to $\xi, V$ is the electronic potential energy surface (PES) along the LAM coordinate (obtained from a relaxed PES scan), and $\hat{H}_{\mathrm{b}}$ is the bath of all other $3 N-7 \mathrm{SAM}$ degrees of freedom ( $N$ denotes the number of atoms in the molecule). $G=\mu^{-1}$ is the inverted reduced mass $(\mu)$ of the LAM. It is being calculated from the generalized tensor of inertia defined as

$$
\mathcal{G}^{-1}=\left(\begin{array}{ll}
\mathcal{I}_{\mathrm{rr}} & \mathbf{I}_{\mathrm{rv}} \\
\mathbf{I}_{\mathrm{rv}}^{\dagger} & I_{\mathrm{vv}}
\end{array}\right)
$$

where $\mathcal{I}_{\text {rr }}=\sum_{k=1}^{N} m_{k}\left(\operatorname{diag}\left(\mathbf{r}_{k} \cdot \mathbf{r}_{k}\right)-\mathbf{r}_{k} \otimes \mathbf{r}_{k}\right)$ is the $3 \times 3$ tensor of inertia, $\mathbf{I}_{\mathrm{rv}}=\sum_{k=1}^{N} m_{k}\left[\mathbf{r}_{k} \times \partial_{\xi} \mathbf{r}_{k}\right]$ is the vector of LAM - rotation interaction, and $I_{\mathrm{vv}}=\sum_{k=1}^{N} m_{k}\left(\partial_{\xi} \mathbf{r}_{k} \cdot \partial_{\xi} \mathbf{r}_{k}\right)$. Here, $m$ and $\mathbf{r}$ denote the atomic masses and positions 1.1

\footnotetext{
$1 \cdot, \times$, and $\otimes$ denote scalar, cross, and outer products of the vectors.
} 
$G$ is the last diagonal element of matrix $\mathcal{G}^{-1}$.

Let $\mathbf{r}=\left(x_{1}, y_{1}, z_{1}, x_{2} \ldots\right)^{\dagger}$ be the $3 N$-sized vector with Cartesian coordinates of atoms, and $\mathbf{m}=\left(m_{1}, m_{1}, m_{1}, m_{2}, \ldots\right)^{\dagger}$ $3 N$-sized vector of atomic masses. Let $\mathbf{R}=\sqrt{\mathbf{m}} \mathbf{r}$ be mass-weighted coordinates of the system ${ }^{2}$ and $\mathcal{F}(\xi)-$ the Wilson's GF-matrix [71] composed of $\mathcal{F}_{i j}=\partial_{R_{i}} \partial_{R_{j}} V=\partial_{r_{i}} \partial_{r_{j}} V / \sqrt{m_{i} m_{j}}$ elements. $\mathbf{R}_{\text {eq }}(\xi)=\sqrt{\mathbf{m}} \mathbf{r}_{\text {eq }}(\xi)$ denotes the equilibrium values of atomic coordinates at the LAM coordinate value $\xi$. Then the harmonic vibrations Hamiltonian $\hat{H}_{\mathrm{b}}$ is given as: 72

$$
\hat{H}_{\mathrm{b}}(\xi)=\frac{1}{2} \hat{\mathbf{P}}_{\mathrm{MWC}}^{2}+\frac{1}{2}\left(\mathbf{R}-\mathbf{R}_{\mathrm{eq}}(\xi)\right)^{\dagger} \mathcal{F}(\xi)\left(\mathbf{R}-\mathbf{R}_{\mathrm{eq}}(\xi)\right)
$$

where $\hat{\mathbf{P}}_{\mathrm{MWC}}=-i \hbar \partial_{\mathbf{R}}$

We can remove translations/rotations of the molecule as a whole, as well as the LAM, from $\mathcal{F}(\xi)$ using matrix $\mathcal{S}(\xi)$. As a result, one can get the harmonic bath GF-matrix $\mathcal{F}_{3 N-7}(\xi)=\mathcal{S}^{\dagger}(\xi) \mathcal{F}(\xi) \mathcal{S}(\xi)$. We chose one of the structures (at $\xi=\xi_{\text {ref }}$ ) as the reference. In our double-well cases, we chose the transition state with $\xi_{\text {ref }}=0$. Then we define the vibrational state of the bath using the normal modes of the reference structure $\left(\mathcal{L}_{\text {ref }}\right)$ given as diagonalizing matrix for $\mathcal{F}_{3 N-7}\left(\xi_{\text {ref }}\right)\left(\mathcal{L}_{\text {ref }}^{\dagger} \mathcal{F}_{3 N-7}\left(\xi_{\text {ref }}\right) \mathcal{L}_{\text {ref }}=\operatorname{diag}\left(\boldsymbol{\Omega}_{\text {ref }}^{2}\right)\right.$, with $\boldsymbol{\Omega}_{\text {ref }}^{2}$ being a vector of squared angular frequencies $)$. 71, 72 This allows us to write $\left(\mathbf{R}-\mathbf{R}_{\text {eq }}(\xi)\right)=\mathcal{L}_{\text {ref }} \mathbf{Q}$, where $\mathbf{Q}$ is the position of the nuclei along the normal modes expressed in mass-weighted coordinates. Note that this assumption requires all the structures to be oriented with respect to the reference coordinates. These operations give the harmonic bath Hamiltonian in the form

$$
\hat{H}_{\mathrm{b}}(\xi)=\underbrace{\frac{1}{2} \sum_{k}\left(\hat{P}_{k}^{2}+\omega_{k}^{2} \hat{Q}_{k}^{2}\right)}_{\hat{H}_{0}}+\underbrace{\frac{1}{2} \sum_{k}\left(\Omega_{k k}^{2}-\omega_{k}^{2}\right) \hat{Q}_{k}^{2}}_{\hat{W}_{1}}+\underbrace{\frac{1}{2} \sum_{k} \sum_{l \neq k} \Omega_{k l}^{2} \hat{Q}_{k} \hat{Q}_{l}}_{\hat{W}_{2}},
$$

where $k$ enumerates normal modes, $\hat{P}_{k}=-i \hbar \partial_{Q_{k}}, \Omega_{k l}^{2}$ are the elements of the matrix $\boldsymbol{\Omega}^{2}(\xi)=\mathcal{L}_{\text {ref }}^{\dagger} \mathcal{F}_{3 N-7}(\xi) \mathcal{L}_{\text {ref }}$, and $\omega_{k}$ are the zeroth approximation frequencies. We can either set them at $\boldsymbol{\Omega}_{\text {ref }}$ values, or allow bath vibrations to relax during the LAM by setting $\omega_{k}$ to the respective diagonal values of $\Omega^{2}(\xi) . \hat{H}_{0}$ denotes the unperturbed vibrational Hamiltonian, and the $\hat{W}_{k}$ are the perturbation operators.

The mass-weighted $\hat{P}_{k} / \hat{Q}_{k}$ (normal modes momentum/position operators) are related to dimension-less operators $\hat{p}_{k}, \hat{q}_{k}$ corresponding to an oscillator with reference frequency $\omega_{k}$ as

$$
\left\{\begin{array}{l}
\hat{p}_{k}=\frac{\hat{P}_{k}}{\sqrt{\hbar \omega_{k}}} \\
\hat{q}_{k}=\sqrt{\frac{\omega_{k}}{\hbar}} \hat{Q}_{k}
\end{array}\right.
$$

We can use them to rewrite the Eq. 3 as

$$
\hat{H}_{\mathrm{b}}(\xi)=\underbrace{\sum_{k} \hbar \omega_{k}\left(\hat{n}+\frac{1}{2}\right)}_{\hat{H}_{0}}+\underbrace{\sum_{k} \frac{\hbar}{2 \omega_{k}}\left(\Omega_{k k}^{2}-\omega_{k}^{2}\right) \hat{q}_{k}^{2}}_{\hat{W}_{1}}+\underbrace{\sum_{k} \sum_{l \neq k} \frac{\hbar \Omega_{k l}^{2}}{2 \sqrt{\omega_{k} \omega_{l}}} \hat{q}_{k} \hat{q}_{l}}_{\hat{W}_{2}}
$$

where $\hat{n}=\hat{a}^{\dagger} \hat{a}$ with $\hat{a}=\frac{\hat{q}_{k}+i \hat{p}_{k}}{\sqrt{2}}$ and $\hat{a}^{\dagger}=\frac{\hat{q}_{k}-i \hat{p}_{k}}{\sqrt{2}}$ (annihilation/creation operators) $3^{3}$

If we consider the bath to be in a chosen vibrational eigenstate $|\mathbf{v}\rangle=\prod_{k}\left|v_{k}\right\rangle$ (with $\mathbf{v}=\left(v_{1}, v_{2}, \ldots\right)$ being a vector of quantum numbers $\left.v_{k}=0,1,2, \ldots\right)$ of the Hamiltonian $\hat{H}_{0}\left(\hat{H}_{0}|\mathbf{v}\rangle=\sum_{k} \hbar \omega_{k}\left(v_{k}+1 / 2\right)|\mathbf{v}\rangle\right)$, then we can apply perturbation theory $(\mathrm{PT})$ to get the expression of the bath energy. Using the secondary quantization form of $\hat{p}_{k}$ and $\hat{q}_{k}$, one can compute the bath Hamiltonian energies.

- $0^{\text {th }}$ order.

$$
E_{\mathbf{v}}^{(0)}=\left\langle\mathbf{v}\left|\hat{H}_{0}\right| \mathbf{v}\right\rangle=\sum_{k} \hbar \omega_{k}\left(v_{k}+\frac{1}{2}\right),
$$

- $1^{\text {st }}$ order correction $\left(\left\langle\mathbf{v}\left|\hat{W}_{2}\right| \mathbf{v}\right\rangle=0\right) 4^{4}$

$$
\delta E_{\mathbf{v}}^{(1)}=\left\langle\mathbf{v}\left|\hat{W}_{1}\right| \mathbf{v}\right\rangle=\sum_{k} \frac{\hbar}{2 \omega_{k}}\left(\Omega_{k k}^{2}-\omega_{k}^{2}\right)\left(v_{k}+\frac{1}{2}\right) .
$$

- $2^{\text {st }}$ order correction: ${ }^{4}$

$$
\delta E_{\mathbf{v}}^{(2.1)}=-\sum_{\mathbf{v}^{\prime} \neq \mathbf{v}} \frac{\left|\left\langle\mathbf{v}^{\prime}\left|\hat{W}_{1}\right| \mathbf{v}\right\rangle\right|^{2}}{E_{\mathbf{v}^{\prime}}^{(0)}-E_{\mathbf{v}}^{(0)}}=-\sum_{k} \frac{\hbar\left(\Omega_{k k}^{2}-\omega_{k}^{2}\right)^{2}}{8 \omega_{k}^{3}}\left(v_{k}+\frac{1}{2}\right)
$$

\footnotetext{
$2 \sqrt{\mathbf{m}}=\left(\sqrt{m_{1}}, \sqrt{m_{2}}, \sqrt{m_{2}}, \sqrt{m_{2}}, \ldots\right)^{\dagger}$.

${ }^{3}$ I.e. $\hat{a}|v\rangle=\sqrt{v}|v-1\rangle, \hat{a}^{\dagger}|v\rangle=\sqrt{v+1}|v+1\rangle$ and $\hat{n}|v\rangle=v|v\rangle$.

${ }^{4}$ Using the fact that $\hat{q}^{2}=\frac{\left(\hat{a}^{\dagger}\right)^{2}+\hat{a}^{2}}{2}+\hat{n}+\frac{1}{2}$.
} 
and

$$
\delta E_{\mathbf{v}}^{(2.2)}=-\sum_{\mathbf{v}^{\prime} \neq \mathbf{v}} \frac{\left|\left\langle\mathbf{v}^{\prime}\left|\hat{W}_{2}\right| \mathbf{v}\right\rangle\right|^{2}}{E_{\mathbf{v}^{\prime}}^{(0)}-E_{\mathbf{v}}^{(0)}}=-\sum_{k} \sum_{l \neq k} \frac{\hbar \Omega_{k l}^{4}}{4 \omega_{k} \omega_{l}} \frac{\omega_{k}\left(v_{l}+\frac{1}{2}\right)-\omega_{l}\left(v_{k}+\frac{1}{2}\right)}{\omega_{k}^{2}-\omega_{l}^{2}}
$$

The resulting energy is:

$$
E_{\mathbf{v}} \approx \underbrace{\sum_{k} \hbar \frac{3 \omega_{k}^{4}+6 \Omega_{k k}^{2} \omega_{k}^{2}-\Omega_{k k}^{4}}{8 \omega_{k}^{3}}\left(v_{k}+\frac{1}{2}\right)}_{E_{\mathbf{v}}^{(0)}+\delta E_{\mathbf{v}}^{(1)}+\delta E_{\mathbf{v}}^{(2.1)}} \underbrace{-\sum_{k} \sum_{l \neq k} \frac{\hbar \Omega_{k l}^{4}}{4 \omega_{k} \omega_{l}} \frac{\omega_{k}\left(v_{l}+\frac{1}{2}\right)-\omega_{l}\left(v_{k}+\frac{1}{2}\right)}{\omega_{k}^{2}-\omega_{l}^{2}}}_{\delta E_{\mathbf{v}}^{(2.2)}}
$$

For the pure LAM excitations we can assume that the other molecular degrees of freedom are at the ground state $(\mathbf{v}=\mathbf{0})$, therefore the energy of the bath is the zero-point vibrational energy (ZPVE) given by an expression

$$
\mathrm{ZPVE}=\sum_{k} \hbar \frac{3 \omega_{k}^{4}+6 \Omega_{k k}^{2} \omega_{k}^{2}-\Omega_{k k}^{4}}{16 \omega_{k}^{3}}-\sum_{k} \sum_{l \neq k} \frac{\hbar \Omega_{k l}^{4}}{8 \omega_{k} \omega_{l}\left(\omega_{k}+\omega_{l}\right)}
$$

If we allow vibrational states relaxation, then $\omega_{k}=\Omega_{k k}$, which gives

$$
\operatorname{ZPVE}(\xi)=\sum_{k} \frac{\hbar \Omega_{k k}(\xi)}{2}-\sum_{k} \sum_{l \neq k} \frac{\hbar \Omega_{k l}^{4}(\xi)}{8 \Omega_{k k}(\xi) \Omega_{l l}(\xi)\left(\Omega_{k k}(\xi)+\Omega_{l l}(\xi)\right)}
$$

By combining Eqs. 1 and 6 the final effective Hamiltonian for LAM looks as the following:

$$
\hat{H}=-\frac{1}{2} \hat{p}_{\xi} G(\xi) \hat{p}_{\xi}+\overbrace{V(\xi)+\operatorname{ZPVE}(\xi)}^{V_{\text {eff }}(\xi)} .
$$

The parameters for this Hamiltonian (effective masses $G(\xi)$ and effective PES $V_{\text {eff }}(\xi)$ ) in this work were computed from relaxed PES scans obtained with Orca 4 using in-house Python scripts. 73 In brief, the script for computing the Hamiltonian parameters works as following.

- The code reads Orca output *.hess files containing vibrational information (molecular geometry, atomic masses, Hessian, etc.).

- The molecular geometries and Hessians are oriented with respect to the reference geometry defined as the molecular structure with the lowest value of $|\xi|$ (the closest to the transition state). The orientation corresponds to the minimum of the functional $\sum_{k=1}^{N} m_{k}\left(\mathcal{R} \mathbf{r}_{k}(\xi)-\mathrm{r}_{\text {ref, }, k}\right)^{2}$, where $\mathcal{R}$ is the rotation matrix.

- The effective masses $\mu=G^{-1}$ are computed as described above. The derivatives $\frac{d \mathbf{r}_{k}}{d \xi}(\xi)$ are computed by fitting the expression $\mathbf{r}_{k}\left(\xi^{\prime}\right) \approx \mathbf{r}_{k}(\xi)+\frac{d \mathbf{r}_{k}}{d \xi}(\xi)\left(\xi^{\prime}-\xi\right)$ to the nearest structures with linear least-squares method.

- The vibrational problem for each structure is solved with exclusion of translations, rotation and LAM direction, given by the $\frac{d \mathbf{r}_{k}}{d \xi}(\xi)$ vector, the spurious imaginary frequencies are also being set to zero. The resulting Hessians are stored.

- The ZPVE correction is computed for each structure by projecting the reference structure normal modes onto the Hessians retrieved in the previous item.

- The obtained dependencies for $\mu(\xi), V(x)$ and $\operatorname{ZPVE}(\xi)$, are extrapolated using cubic splines to a fine uniform grid.

The resulting eigenvalue problem is solved using the discrete variable representation (DVR) method, in particular, sinc-DVR. 74

\section{Results and discussion}

\subsection{Proof of concept: ammonia}

To test the performance of the implementation, we chose the simplest case of LAM: an inversion motion in ammonia $\left(\mathrm{NH}_{3}\right)$. The LAM coordinate was defined as

$$
\xi_{\mathrm{NH}_{3}}=\operatorname{sign}\left(\mathbf{r}_{\mathrm{NH} 1} \cdot\left[\mathbf{r}_{\mathrm{NH} 2} \times \mathbf{r}_{\mathrm{NH} 3}\right]\right) \cdot\left(\angle_{\mathrm{H} 1 \mathrm{NH} 2}+\angle_{\mathrm{H} 1 \mathrm{NH} 3}+\angle_{\mathrm{H} 2 \mathrm{NH} 3}-360^{\circ}\right),
$$

where $\mathbf{r}_{\mathrm{NH} n}$ denotes a position vector of the hydrogen number $n$ with nitrogen as an origin, and $\angle_{\mathrm{H} n \mathrm{NH} m}$ denotes the valence angle $\mathrm{H} n-\mathrm{N}-\mathrm{H} m$ value (in degrees). The sign of the oriented volume defined by the vectors $\mathbf{r}_{\mathrm{NH} n}$ is needed to discriminate between normal and inverted ammonia, because valence angles are not sensitive to that. 

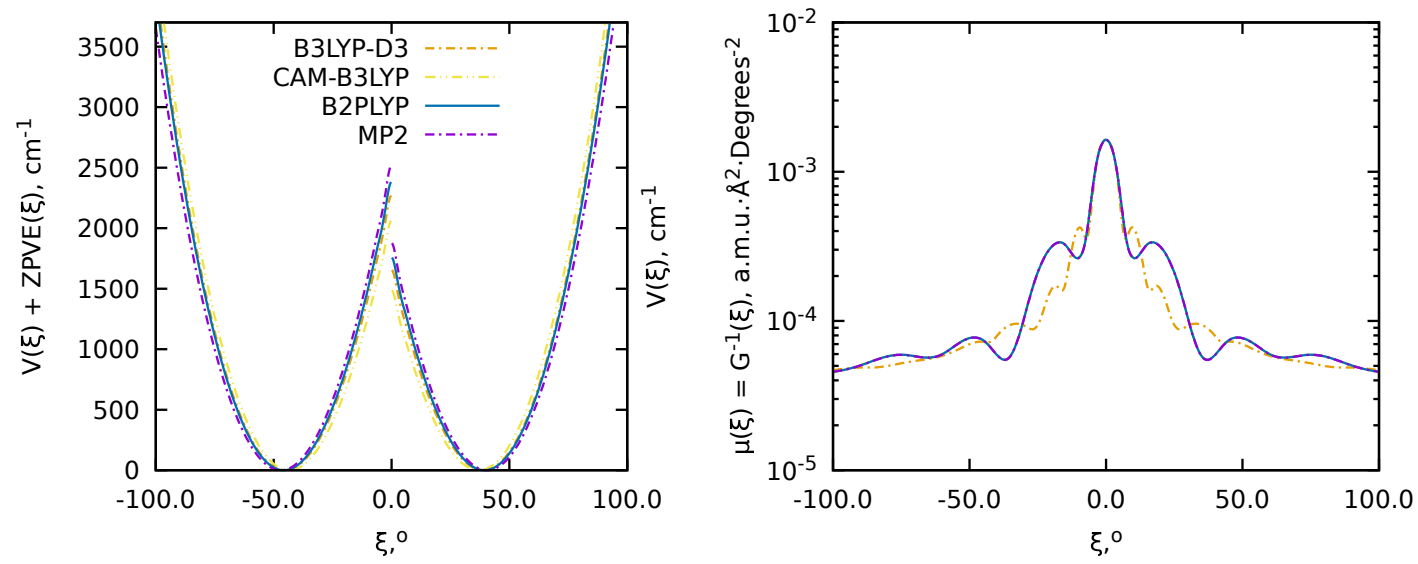

Figure 2: Effective $(V(\xi)+\operatorname{ZPVE}(\xi))$ and normal $(V(\xi))$ potential energy surfaces, and effective masses $\mu(\xi)$ of inversion for the parent isotopologue of ammonia computed at various levels of theory with def2-TZVPP basis set. The reaction coordinate $\xi$ is given by Eq. 8 .

Table 1: Vibrational state energies for the inversion of ammonia and some of its isotopologues. Experimental values are taken from Ref. 6], theoretical values are computed with a scan obtained at the CAM-B3LYP/def2-TZVPP level of theory. All the values are in $\mathrm{cm}^{-1}$.

\begin{tabular}{crrrrrrrr}
\hline \hline State & \multicolumn{2}{c}{$\mathrm{NH}_{3}$} & \multicolumn{2}{c}{$\mathrm{ND}_{3}$} & \multicolumn{2}{c}{${ }^{15} \mathrm{ND}_{3}$} & $\mathrm{NT}_{3}$ \\
\cline { 2 - 8 } & Exp. & Theor. & Exp. & Theor. & Exp. & Theor. & Exp. & Theor. \\
\hline $0^{+}$ & 0 & 0 & 0 & 0 & 0 & 0 & 0 & 0 \\
$0^{-}$ & 0.76 & 0.21 & 0.05 & 0.02 & 0.05 & 0.02 & 0.01 & 0.004 \\
$1^{+}$ & 928.5 & 941.2 & 745.6 & 753.3 & 739.5 & 748.0 & 656.4 & 667.8 \\
$1^{-}$ & 962.9 & 967.6 & 749.2 & 757.5 & 742.8 & 751.9 & 656.4 & 669.1 \\
$2^{+}$ & 1591.2 & 1508.8 & 1359.0 & 1175.6 & & & & \\
$2^{-}$ & 1870.9 & 1588.6 & 1429.0 & 1199.1 & & & & \\
$3^{+}$ & 2369.3 & 2299.6 & 1830.0 & 1744.9 & & & & \\
$3^{-}$ & 2876.1 & 2737.1 & 2106.6 & 1919.5 & & & & \\
\hline \hline
\end{tabular}

The 1D PES scans were performed at B3LYP-D3BJ, CAM-B3LYP, MP2 and B2PLYP levels of theory. The resulting parameters of the LAM Hamiltonian (Eq. 7) are given in Figure 2. All the methods performed similar to each other. The energy levels of ammonia and some of its isotopologues obtained with CAM-B3LYP are given in Table 1. Concerning simplicity of the calculation, the results are very similar to the experimental values. The values of other methods can be found in the SI. The results for ammonia confirm the workability of the code, which justify testing of the approach on larger molecules.

\subsection{Proton transfer in malonaldehyde (MA) and formic acid dimer (FAD)}

\subsubsection{Tunneling splittings in the parent species}

The PESs for proton transfer in MA and FAD were computed at various levels of theory, using both post-Hartree Fock methods (MP2 and SCS-MP2) and different DFT functionals from different steps of Perdew's ladder:75] GGA (PBE and BLYP), hybrid (B3LYP and PBE0), range-separated hybrid (CAM-B3LYP), and double hybrid (B2PLYP and $\mathrm{mPW}$ 2PLYP). FAD is a dimer; therefore we took into account possible missing dispersion interactions in case of GGA and hybrid functionals via D3BJ correction. The same correction was also tested together with one of the double-hybrid functionals (B2PLYP).

The proton transfer coordinates were as following. In case of MA:

$$
\xi=\frac{r(\mathrm{O} \cdots \mathrm{H})-r(\mathrm{O}-\mathrm{H})}{2}
$$

and for FAD as

$$
\xi=\frac{r\left(\mathrm{O}-\mathrm{H}_{\mathrm{a}}\right)+r\left(\mathrm{O}-\mathrm{H}_{\mathrm{b}}\right)-r\left(\mathrm{O} \cdots \mathrm{H}_{\mathrm{a}}\right)-r\left(\mathrm{O} \cdots \mathrm{H}_{\mathrm{b}}\right)}{2 \sqrt{2}} .
$$

The obtained Hamiltonian parameters (PES and effective masses) for these calculations are plotted in Figure 3 the computed tunneling splittings between ground vibrational states of different parity $\left(0^{+} / 0^{-}\right)$are given in Table 2 As it can be seen from these results, most of the tested methods give reasonable estimates of the tunneling splittings compared to the experimental values determined in Refs. [9 11]. The pure GGA functionals with dispersion corrections 

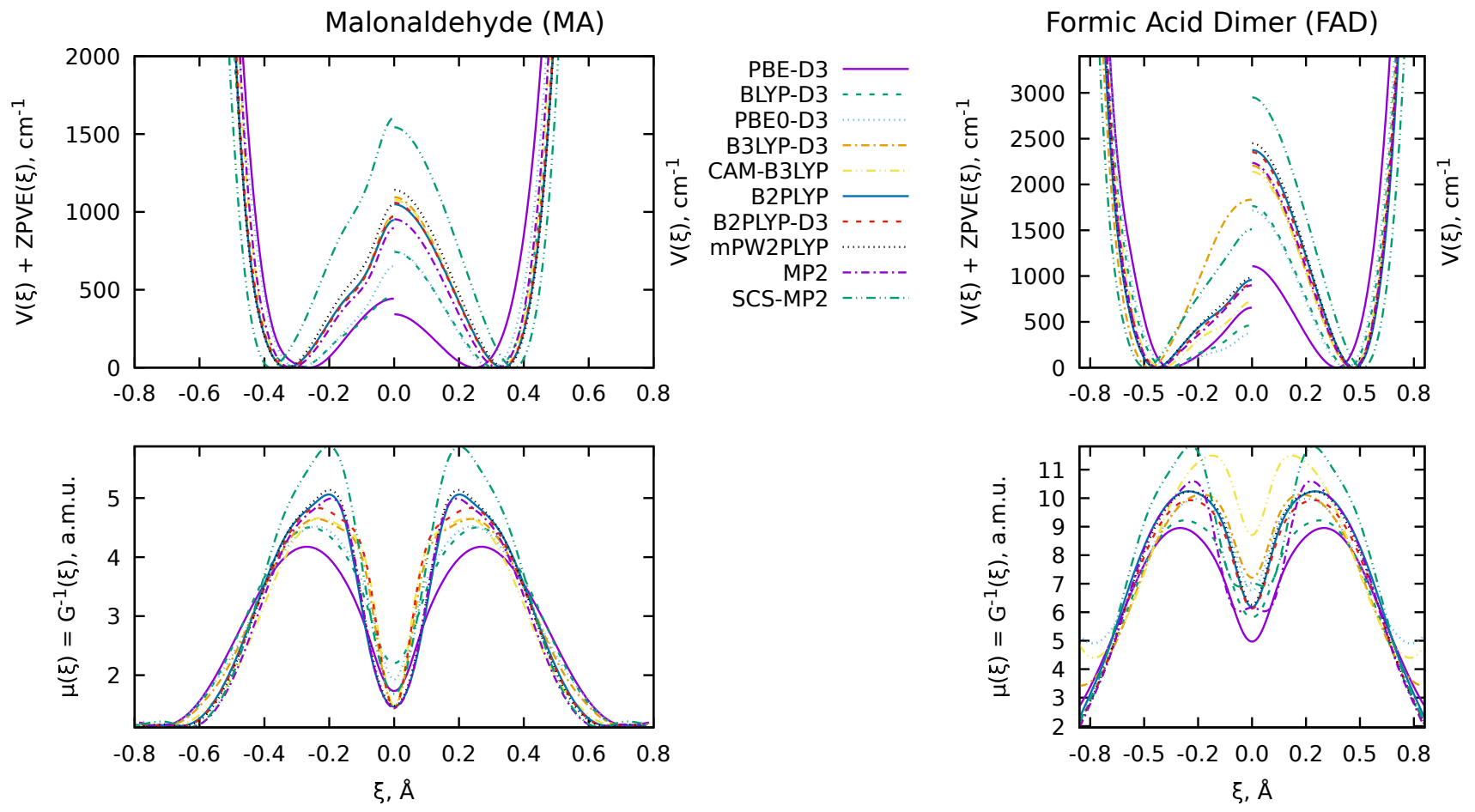

Figure 3: Effective $(V(\xi)+\mathrm{ZPVE}(\xi))$ and normal $(V(\xi))$ potential energy surfaces and effective masses $\mu(\xi)$ of inversion for the parent isotopologues of MA and FAD computed at various levels of theory with def2-TZVPP basis set. The reaction coordinates $\xi$ for $\mathrm{MA}$ and $\mathrm{FAD}$ are given by Eqs. 9 and 10 , respectively.

(PBE-D3BJ and BLYP-D3BJ), as well as the hybrid PBE0-D3BJ, perform not well due to underestimation of the proton transfer barriers. Double hybrids (especially B2PLYP without D3BJ), MP2, and CAM-B3LYP work stable for both MA and FAD. B3LYP-D3BJ performs well for MA, but in case of FAD it underestimates the barrier height, which might be a result of the intramolecular nature of the bonding forces in this complex. SCS-MP2 showed the worst performance due to overestimation of the proton transfer barriers.

\subsubsection{Tunneling splittings in the MA isotopologues}

Similar to ammonia, MA has experimental information available on the tunneling splittings in its isotopologues. 7, , 8, These parameters were calculated for MA-D8, MA-D6 and MA-D6D8 isotopologues at B3LYP-D3BJ, CAM-B3LYP, B2PLYP and MP2 levels of theory, with def2-TZVPP basis set. The results are given in Table 3 . The value for the MA-D8 conformer is close to the parent isotopologue. This is expected, since the hydrogen number 8 is on the opposite side of the molecule with respect to the proton transfer $\mathrm{O}-\mathrm{H} \cdots \mathrm{O}$ fragment. The MA-D6 and MA-D6D8 isotopologues, as expected, show lower tunneling splitting values, since hydrogen number 6 is the one being transferred between the oxygens during the LAM. However, the agreement with experimental values is worse compared to the parent MA. This is not observed in the case of $\mathrm{NH}_{3}$, where all the vibrational levels for the substituted isotopologues are predicted with approximately the same level of accuracy as for the parent species.

The most probable explanation to that is breaking of the adiabaticity assumed in the Hamiltonian given in Eq. 2 . Isotopic substitution that leads to an increase of the atomic masses with respect to the parent isotopologue (deuteration, ${ }^{13} \mathrm{C}$ and ${ }^{15} \mathrm{~N}$ substitution) reduces the vibrational frequencies of the SAM in the bath. This can make these SAM susceptible to vibrational excitation by LAM because of the reduced energy difference between effective potential surfaces. MA has low-energy vibrational modes, which might lead to this adiabaticity violation. This is supported by the plots of the density of the LAM vibrational manifolds given as $V_{\mathbf{v}}(\xi)=V(\xi)+E_{\mathbf{v}}(\xi)$ with vibrational energy of the SAM defined by Eq. 5 (see Figure 4). In case of $\mathrm{NH}_{3}, \mathrm{ND}_{3}$, and the parent MA isotopologue, the effective potential energy $V_{\mathbf{0}}(\xi)=\mathrm{ZPVE}(\xi)$ is well separated from the excited vibrational manifolds. However, in the case of MA-D6, the other manifolds get close to the $V_{\mathbf{0}}(\xi)$ at the positions $\xi$, which are populated in the ground vibrational state. This means that multiple vibrational manifolds might be needed for consideration in order to get a good description of the LAM ground vibrational state of the MA-D6.

\subsubsection{Complete basis set (CBS) extrapolation of the tunneling splittings}

Karlsruhe basis set series 62 can be used for the complete basis set (CBS) extrapolation, [76, 77] therefore a general possibility of an extrapolation to the complete basis set (CBS) limit for the tunneling splitting values was investigated. For this purpose, we have computed the $0^{+} / 0^{-}$tunneling splittings in MA and FAD using def2-nVP and def2-nVPD basis set series ( $\mathrm{n}=\mathrm{S}, \mathrm{TZ}, \mathrm{QZ}$, which corresponds to cardinal number $\zeta=2,3,4$, respectively) with the B3LYP- 
Table 2: $0^{+} / 0^{-}$tunneling splittings for proton transfer motions in MA and FAD obtained with def2-TZVPP basis sets. Experimental values were taken from Ref. [9] for MA and from Refs. [10, 11] for FAD. All the values are in $\mathrm{cm}^{-1}$.

\begin{tabular}{ccc}
\hline \hline Method & MA & FAD \\
\hline PBE-D3BJ & 89.0 & 1.591 \\
BLYP-D3BJ & 59.0 & 2.370 \\
PBE0-D3BJ & 51.1 & 3.989 \\
B3LYP-D3BJ & 17.4 & 0.115 \\
CAM-B3LYP & 19.7 & 0.073 \\
B2PLYP & 20.3 & 0.019 \\
B2PLYP-D3BJ & 17.2 & 0.035 \\
mPW2PLYP & 14.8 & 0.014 \\
MP2 & 28.8 & 0.059 \\
SCS-MP2 & 1.5 & $1 \cdot 10^{-4}$ \\
\hline Experiment & $21.5831383(6)$ & $0.01117 / 0.011367(92)$ \\
\hline \hline
\end{tabular}

Table 3: Tunneling splittings for proton transfer in MA and some of its isotopologues. See Figure 1] and Refs. [7, 8] for enumeration of the atoms. All the values are in $\mathrm{cm}^{-1}$.

\begin{tabular}{lcrrrr}
\hline \hline Isotopologue & Exp. & B3LYP-D3BJ & CAM-B3LYP & B2PLYP & MP2 \\
\hline MA-parent & $21.5831383(6)$ & 17.3 & 19.6 & 20.3 & 28.8 \\
MA-D8 & $26(10)$ & 17.0 & 19.4 & 20.0 & 28.4 \\
MA-D6 & $2.915(4)$ & 9.2 & 7.8 & 7.2 & 10.8 \\
MA-D6D8 & $2.88360(2)$ & 9.0 & 7.6 & 7.1 & 10.6 \\
\hline \hline
\end{tabular}

D3BJ functional. The tunneling splittings were then approximated by an exponential fitting formula $\Delta E_{0^{+}} / 0^{-}(\zeta)=$ $\Delta E_{0^{+} / 0^{-}}^{\mathrm{CBS}}+A \cdot \exp (-\alpha \cdot \zeta)$, where $\Delta E_{0^{+} / 0^{-}}$denote the value of the splitting obtained at different basis sets and the CBS-extrapolated value. Due to smaller values of the tunneling splitting of FAD, which give rise to larger numerical instabilities, the $\alpha$ parameters (1.5 for def2-nVP and 1.2 for def2-nVPD) for the CBS extrapolation for FAD were taken from the MA fits. The results are shown in Fig. 5. Both def2-nVP and def2-nVPD basis set series converge to similar values of $\Delta E_{0^{+} / 0^{-}}^{\mathrm{CBS}}$. The used basis series consist only of three basis sets, therefore we could not make a proper estimation of the CBS extrapolation errors in each serie, however, the similar trends for the tunneling splittings are clearly visible. The usage of the $\alpha$ parameter obtained from MA for FAD tunneling splittings indicates a transferability of the trends from one molecular system to another. Thus, we conclude that the CBS extrapolation is also, in principle, possible for such complicated parameters as tunneling splittings.

\subsubsection{Reaction coordinate choice invariance}

The proposed approach explicitly relies on the reaction coordinate definition: for calculations of both reduced mass of the motion and of the SAM ZPVE correction. Although, by construction, the Schödinger equation solutions produced by any of the properly chosen coordinates (i.e. by those, that can map a transition from one minimum to another through the vicinity of the transition state) should be the same. However, the numerical implementation of the methods might be rather sensitive to the coordinate choice. In order to check this stability of the algorithm we have performed the same type of calculation for MA at B2PLYP/def2-TZVPP level of theory with ill-defined reactions coordinates (see Fig. 1 for atomic enumeration):

$$
\xi_{\mathrm{CH}}=\frac{r(\mathrm{C} 2 \ldots \mathrm{H} 6)-r(\mathrm{C} 4 \ldots \mathrm{H} 6)}{2}
$$

and

$$
\xi_{\mathrm{HH}}=\frac{r(\mathrm{H} 7 \ldots \mathrm{H} 6)-r(\mathrm{H} 9 \ldots \mathrm{H} 6)}{2} .
$$

The value computed with the initial reaction coordinate (Eq. 9) was $20.3 \mathrm{~cm}^{-1}$ (see Table 2). By replacing the $\xi$ to $\xi_{\mathrm{CH}}$ and $\xi_{\mathrm{HH}}$ the result did not change significantly: 19.9 and $20.1 \mathrm{~cm}^{-1}$, respectively. Thus, we conclude that the implemented method is rather stable with respect to the reaction coordinate definition.

\subsubsection{Calculation using the composite approaches}

The additional test of performace was done for the composite quantum chemical calculations B//A, where A indicates a computationally cheap quantum chemical method, that is being used as a source of relaxed PES scan and harmonic vibrational frequencies, and B is the high accuracy level of theory, that provides accurate single point energies. In order to check the possibility of such schemes, we used the relaxed PES scan done at B3LYP-D3BJ/def2-TZVPP level of theory, and computed single point energies at these geometries using the MP2, B2PLYP, and ae-CCSD(T) levels 

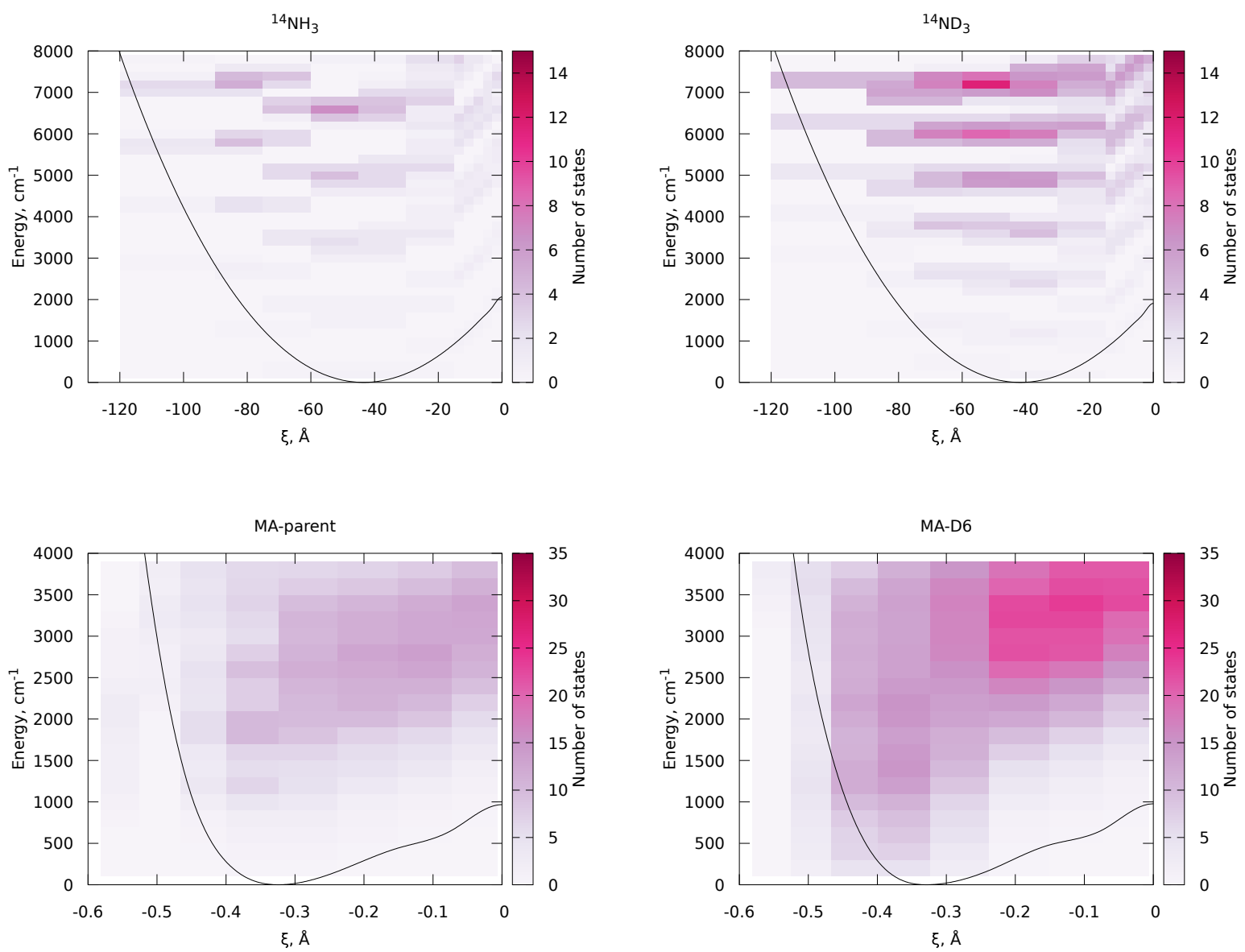

Figure 4: Number of vibrational states $V_{\mathbf{v}}(\xi)=V(\xi)+E_{\mathbf{v}}(\xi)$ with vibrational energy of the SAM defined by Eq. 5. The black line shows the effective potential $V_{\mathbf{0}}(\xi)=\mathrm{ZPVE}(\xi)$ (Eq. 6) for ammonia $\left(\mathrm{NH}_{3}\right)$, its fully deuterated isotopologue $\left(\mathrm{ND}_{3}\right)$, MA, and MA-D6 isotopologue (see Fig. 1 for enumeration). Reaction coordinates $\xi$ for ammonia and MA are given by Eqs. 8 and 9 , respectively.

of theory with the def2-TZVPP basis set. The computed tunneling splittings are given in Table 4 . As one can see, all composite schemes gave as reasonable estimates for the splittings, as the single method calculations. However, the scheme employing the most accurate quantum chemical approximation of all tested (ae-CCSD(T)/def2-TZVPP) gave the splitting 15 wavenumbers lower than the expected value. Such decrease of the value might be caused by a different local shape of the PES at this approximation with respect to the B3LYP-D3BJ/def2-TZVPP. This discrepancy requires additional investigation, which is out of the scope of the current manuscript. Nevertheless, we can conclude that the proposed method for computation of the tunneling splittings should also be applicable with the composite quantum chemical calculations. 

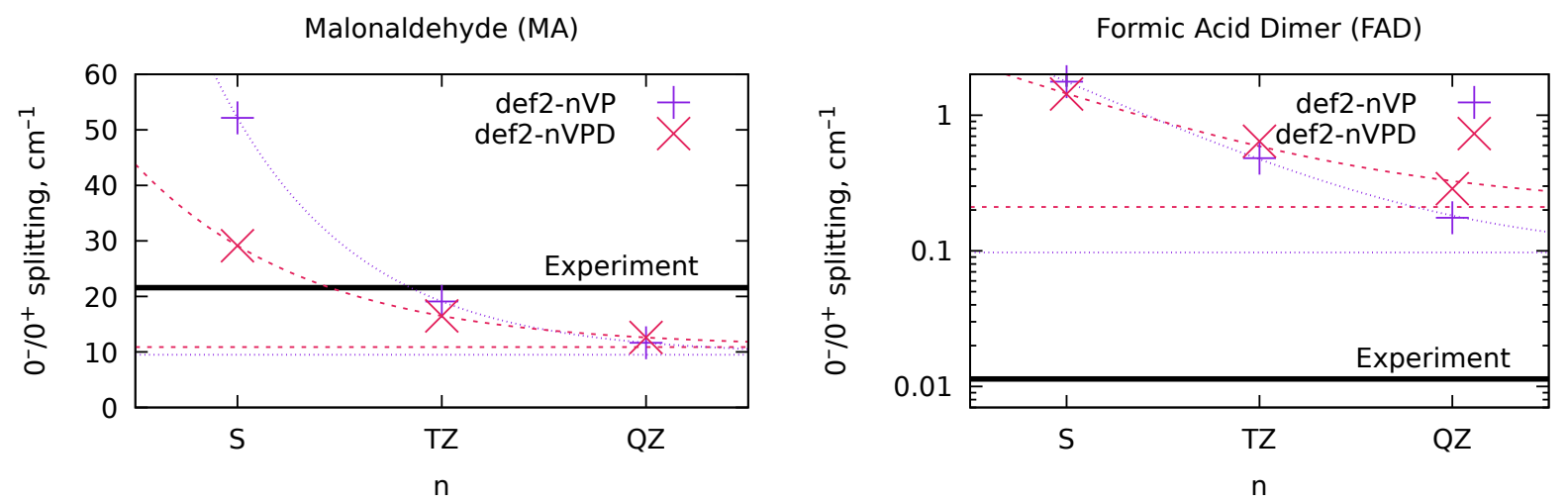

Figure 5: $0^{+} / 0^{-}$tunneling splittings for proton transfer motion in MA and FAD molecules at B3LYP-D3BJ/def2-nVP and B3LYP-D3BJ/def2-nVPD levels of theory, and the complete basis set (CBS) extrapolation of these dependencies (horizontal dashed lines). Solid black horizontal lines denote the experimental values.

Table 4: Theoretical $0^{+} / 0^{-}$tunneling splittings for proton transfer motions in MA at different composite methods. The calculations have used the def2-TZVPP basis set. All the values are in $\mathrm{cm}^{-1}$.

\begin{tabular}{lr}
\hline \hline Method & Splitting \\
\hline B3LYP-D3BJ & 17.4 \\
MP2 & 28.8 \\
B2PLYP & 20.3 \\
MP2//B3LYP-D3BJ & 21.4 \\
B2PLYP//B3LYP-D3BJ & 17.6 \\
ae-CCSD(T)//B3LYP-D3BJ & 7.0 \\
\hline \hline
\end{tabular}

\section{Conclusion}

We have introduced, implemented, and tested an approach for computing the tunneling splittings for one-dimensional large amplitude motions (LAMs). The computational scheme is based on the adiabatic separation of LAM from the small amplitude motions, which are treated as a vibrational bath. The bath zero-point vibrational energy (ZPVE) is added to the electronic potential energy surface (PES) to yield an effective PES. Combined with an effective mass the effective PES produces a one-dimensional Schrödinger equation, and the solutions of the latter allow for determination of the tunneling splitting caused by the LAM between equivalent minima.

The described approach has been shown to work in cases of three model LAMs: nitrogen inversion in ammonia, single proton transfer in MA, and double proton transfer in FAD. Most of the tested methods (B3LYP-D3BJ, CAMB3LYP, B2PLYP, mPW2PLYP, B2PLYP-D3BJ, and MP2) performed reasonably well and thus can be recommended for general usage. However, the pure GGA functionals tested (PBE-D3BJ and BLYP-D3BJ), as well as the PBE0D3BJ and SCS-MP2 methods give splitting values that are quite far away from the experimental values and thus should be avoided in the calculations of the proton transfer tunneling splittings. The methodology with certain limitations was found to be applicable for isotopic substitution: an applicability condition is that the adiabatic separation of the LAM from SAM should remain in the isotopologues. We have also demonstrated a possibility of the CBS extrapolation for the tunneling splittings the of the usage of the composite quantum chemical methods, and the invariance of the calculated spliting with respect to the reaction coordinate choice. In conclusion, the implemented approach can be routinely applied for calculations of the tunneling splittings for the molecules and molecular clusters investigated with MW and MMW spectroscopy.

\section{$5 \quad$ Data availability}

The Python scripts used for calculation of Hamiltonian parameters can be downloaded from the repository (https: //stash.desy.de/projects/MOLINC).73. The quantum-chemical calculations needed to reproduce the results, the results themselves, and raw data for the plots of this paper can be obtained from a SI given by *.zip archive (60 $\mathrm{MB})$.

\section{References}

[1] Per-Olov Löwdin. Proton tunneling in dna and its biological implications. Rev. Mod. Phys., 35:724-732, Jul 1963. doi: 10.1103/RevModPhys.35.724. URL https ://link.aps.org/doi/10.1103/RevModPhys.35.724. 
[2] David M. Leitner, Martina Havenith, and Martin Gruebele. Biomolecule large-amplitude motion and solvation dynamics: modelling and probes from thz to x-rays. International Reviews in Physical Chemistry, 25(4):553-582, 2006. doi: 10.1080/01442350600862117. URL https://doi.org/10.1080/01442350600862117.

[3] K. Giese, M. Petković, H. Naundorf, and O. Kühn. Multidimensional quantum dynamics and infrared spectroscopy of hydrogen bonds. Physics Reports, 430(4):211 - 276, 2006. ISSN 0370-1573. doi: https://doi.org/10.1016/j. physrep.2006.04.005. URL http://www.sciencedirect.com/science/article/pii/S0370157306001608.

[4] Denis S. Tikhonov, Anatolii N. Rykov, Olga E. Grikina, and Leonid S. Khaikin. Gas phase equilibrium structure of histamine. Phys. Chem. Chem. Phys., 18:6092-6102, 2016. doi: 10.1039/C5CP07719B. URL http://dx.doi. org/10.1039/C5CP07719B.

[5] Ruby Srivastava. The role of proton transfer on mutations. Frontiers in Chemistry, 7:536, 2019. ISSN $2296-2646$. doi: 10.3389/fchem.2019.00536. URL https://www.frontiersin.org/article/10.3389/fchem.2019.00536.

[6] V Špirko and W.P Kraemer. Anharmonic potential function and effective geometries for the nh3 molecule. Journal of Molecular Spectroscopy, 133(2):331 - 344, 1989. ISSN 0022-2852. doi: https://doi.org/10.1016/0022-2852(89) 90196-3. URL http://www.sciencedirect.com/science/article/pii/0022285289901963.

[7] Steven L. Baughcum, Richard W. Duerst, Walter F. Rowe, Zuzana Smith, and E. Bright Wilson. Microwave spectroscopic study of malonaldehyde (3-hydroxy-2-propenal). 2. structure, dipole moment, and tunneling. Journal of the American Chemical Society, 103(21):6296-6303, 1981. doi: 10.1021/ja00411a005. URL https://doi.org/10.1021/ja00411a005.

[8] Steven L. Baughcum, Zuzana Smith, E. Bright Wilson, and Richard W. Duerst. Microwave spectroscopic study of malonaldehyde. 3. vibration-rotation interaction and one-dimensional model for proton tunneling. Journal of the American Chemical Society, 106(8):2260-2265, 1984. doi: 10.1021/ja00320a007. URL https://doi .org/10. $1021 / \mathrm{ja00320a007}$

[9] Takeshi Baba, Takehiko Tanaka, Isamu Morino, Koichi M. T. Yamada, and Keiichi Tanaka. Detection of the tunneling-rotation transitions of malonaldehyde in the submillimeter-wave region. The Journal of Chemical Physics, 110(9):4131-4133, 1999. doi: 10.1063/1.478296. URL https://doi.org/10.1063/1.478296

[10] Yuluan Zhang, Wenguang Li, Wei Luo, Yu Zhu, and Chuanxi Duan. High resolution jet-cooled infrared absorption spectra of (hcooh)2, (hcood)2, and hcooh-hcood complexes in $7.2 \mu \mathrm{m}$ region. The Journal of Chemical Physics, 146(24):244306, 2017. doi: 10.1063/1.4989863. URL https://doi.org/10.1063/1.4989863

[11] Weixing Li, Luca Evangelisti, Qian Gou, Walther Caminati, and Rolf Meyer. The barrier to proton transfer in the dimer of formic acid: A pure rotational study. Angewandte Chemie International Edition, 58(3):859-865, 2019. doi: 10.1002/anie.201812754. URL https://onlinelibrary.wiley.com/doi/abs/10.1002/anie.201812754.

[12] Ha Vinh Lam Nguyen and Isabelle Kleiner. Understanding (coupled) large amplitude motions: the interplay of microwave spectroscopy, spectral modeling, and quantum chemistry. Physical Sciences Reviews, 2020. doi: doi:10.1515/psr-2020-0037. URL https://doi.org/10.1515/psr-2020-0037.

[13] Cristóbal Pérez, Matt T. Muckle, Daniel P. Zaleski, Nathan A. Seifert, Berhane Temelso, George C. Shields, Zbigniew Kisiel, and Brooks H. Pate. Structures of cage, prism, and book isomers of water hexamer from broadband rotational spectroscopy. Science, 336(6083):897-901, 2012. ISSN 0036-8075. doi: 10.1126/science. 1220574. URL https://science.sciencemag.org/content/336/6083/897.

[14] Cristóbal Pérez, Amanda L. Steber, Anouk M. Rijs, Berhane Temelso, George C. Shields, Juan Carlos Lopez, Zbigniew Kisiel, and Melanie Schnell. Corannulene and its complex with water: a tiny cup of water. Phys. Chem. Chem. Phys., 19:14214-14223, 2017. doi: 10.1039/C7CP01506B. URL http://dx.doi.org/10.1039/ C7CP01506B.

[15] Melanie Schnell and Jens-Uwe Grabow. Multidimensional large-amplitude motion: Revealing concurrent tunneling pathways in molecules with several internal rotors. Angewandte Chemie International Edition, 45(21):3465-3470, 2006. doi: https://doi.org/10.1002/anie.200600201. URL https://onlinelibrary.wiley.com/doi/abs/10. 1002/anie.200600201.

[16] M. Fatima, D. Maué, C. Pérez, D. S. Tikhonov, D. Bernhard, A. Stamm, C. Medcraft, M. Gerhards, and M. Schnell. Structures and internal dynamics of diphenylether and its aggregates with water. Phys. Chem. Chem. Phys., 22:27966-27978, 2020. doi: 10.1039/D0CP04104A. URL http://dx.doi.org/10.1039/D0CP04104A.

[17] Rizalina Tama Saragi, Marcos Juanes, Cristóbal Pérez, Pablo Pinacho, Denis S. Tikhonov, Walther Caminati, Melanie Schnell, and Alberto Lesarri. Switching hydrogen bonding to $\pi$-stacking: The thiophenol dimer and trimer. The Journal of Physical Chemistry Letters, 12(5):1367-1373, 2021. doi: 10.1021/acs.jpclett.0c03797. URL https://doi.org/10.1021/acs . jpclett.0c03797. 
[18] Yves Marechal and Andrzej Witkowski. Infrared spectra of h-bonded systems. The Journal of Chemical Physics, 48(8):3697-3705, 1968. doi: 10.1063/1.1669673. URL https://doi.org/10.1063/1.1669673.

[19] Hao Liu, Jianwei Cao, and Wensheng Bian. Double proton transfer in the dimer of formic acid: An efficient quantum mechanical scheme. Frontiers in Chemistry, 7:676, 2019. ISSN 2296-2646. doi: 10.3389/fchem.2019. 00676. URL https://www.frontiersin.org/article/10.3389/fchem.2019.00676

[20] Markus Ortlieb and Martina Havenith. Proton transfer in (hcooh)2: An ir high-resolution spectroscopic study of the antisymmetric c-o stretch. The Journal of Physical Chemistry A, 111(31):7355-7363, 2007. doi: 10.1021/ jp070763+. URL https://doi.org/10.1021/jp070763+. PMID: 17552500.

[21] Özgür Birer and Martina Havenith. High-resolution infrared spectroscopy of the formic acid dimer. Annual Review of Physical Chemistry, 60(1):263-275, 2009. doi: 10.1146/annurev.physchem.040808.090431. URL https: //doi.org/10.1146/annurev.physchem.040808.090431. PMID: 18999993.

[22] D. Babić, S.D. Bosanac, and N. Došlić. Proton transfer in malonaldehyde: a model three-dimensional study. Chemical Physics Letters, 358(3):337 - 343, 2002. ISSN 0009-2614. doi: https://doi.org/10.1016/S0009-2614(02) 00636-X. URL http://www.sciencedirect.com/science/article/pii/S000926140200636X.

[23] Ivana Matanović, Nadja Došlić, and Bruce R. Johnson. Generalized approximation to the reaction path: The formic acid dimer case. The Journal of Chemical Physics, 128(8):084103, 2008. doi: 10.1063/1.2833978. URL https://doi.org/10.1063/1.2833978.

[24] Mikhail V. Vener, Oliver Kühn, and Joel M. Bowman. Vibrational spectrum of the formic acid dimer in the oh stretch region. a model 3d study. Chemical Physics Letters, 349(5):562 - 570, 2001. ISSN 00092614. doi: https://doi.org/10.1016/S0009-2614(01)01248-9. URL http://www.sciencedirect.com/science/ article/pii/S0009261401012489.

[25] George L. Barnes and Edwin L. Sibert. The effects of asymmetric motions on the tunneling splittings in formic acid dimer. The Journal of Chemical Physics, 129(16):164317, 2008. doi: 10.1063/1.3000102. URL https: //doi.org/10.1063/1.3000102.

[26] Yonggang Yang and Markus Meuwly. A generalized reactive force field for nonlinear hydrogen bonds: Hydrogen dynamics and transfer in malonaldehyde. The Journal of Chemical Physics, 133(6):064503, 2010. doi: 10.1063/ 1.3447701. URL https://doi.org/10.1063/1.3447701.

[27] George L. Barnes, Shane M. Squires, and Edwin L. Sibert. Symmetric double proton tunneling in formic acid dimer: A diabatic basis approach. The Journal of Physical Chemistry B, 112(2):595-603, 2008. doi: 10.1021/ jp075376e. URL https://doi.org/10.1021/jp075376e. PMID: 18004835.

[28] Feng Wu, Yinghui Ren, and Wensheng Bian. The hydrogen tunneling splitting in malonaldehyde: A fulldimensional time-independent quantum mechanical method. The Journal of Chemical Physics, 145(7):074309, 2016. doi: 10.1063/1.4960789. URL https://doi.org/10.1063/1.4960789.

[29] Tomoyuki Hayashi and Shaul Mukamel. Multidimensional infrared signatures of intramolecular hydrogen bonding in malonaldehyde. The Journal of Physical Chemistry A, 107(43):9113-9131, 2003. doi: 10.1021/jp030626m. URL https://doi.org/10.1021/jp030626m

[30] Thorsten Hammer and Uwe Manthe. Intramolecular proton transfer in malonaldehyde: Accurate multilayer multi-configurational time-dependent hartree calculations. The Journal of Chemical Physics, 134(22):224305, 2011. doi: 10.1063/1.3598110. URL https://doi.org/10.1063/1.3598110.

[31] Maurício Coutinho-Neto, Alexandra Viel, and Uwe Manthe. The ground state tunneling splitting of malonaldehyde: Accurate full dimensional quantum dynamics calculations. The Journal of Chemical Physics, 121(19): 9207-9210, 2004. doi: 10.1063/1.1814356. URL https://doi.org/10.1063/1.1814356.

[32] Chen Qu and Joel M. Bowman. An ab initio potential energy surface for the formic acid dimer: zero-point energy, selected anharmonic fundamental energies, and ground-state tunneling splitting calculated in relaxed 1-4-mode subspaces. Phys. Chem. Chem. Phys., 18:24835-24840, 2016. doi: 10.1039/C6CP03073D. URL http: //dx.doi.org/10.1039/C6CP03073D.

[33] Yimin Wang, Bastiaan J. Braams, Joel M. Bowman, Stuart Carter, and David P. Tew. Full-dimensional quantum calculations of ground-state tunneling splitting of malonaldehyde using an accurate ab initio potential energy surface. The Journal of Chemical Physics, 128(22):224314, 2008. doi: 10.1063/1.2937732. URL https://doi. org/10.1063/1.2937732.

[34] Alexandra Viel, Maurício D. Coutinho-Neto, and Uwe Manthe. The ground state tunneling splitting and the zero point energy of malonaldehyde: A quantum monte carlo determination. The Journal of Chemical Physics, 126 (2):024308, 2007. doi: 10.1063/1.2406074. URL https://doi.org/10.1063/1.2406074. 
[35] Jeremy O. Richardson and Stuart C. Althorpe. Ring-polymer instanton method for calculating tunneling splittings. The Journal of Chemical Physics, 134(5):054109, 2011. doi: 10.1063/1.3530589. URL https: //doi.org/10.1063/1.3530589.

[36] Gennady Mil'nikov and Hiroki Nakamura. Tunneling splitting and decay of metastable states in polyatomic molecules: invariant instanton theory. Phys. Chem. Chem. Phys., 10:1374-1393, 2008. doi: 10.1039/B712988B. URL http://dx.doi.org/10.1039/B712988B.

[37] G. V. Mil'nikov, O. Kühn, and H. Nakamura. Ground-state and vibrationally assisted tunneling in the formic acid dimer. The Journal of Chemical Physics, 123(7):074308, 2005. doi: 10.1063/1.2000257. URL https: //doi.org/10.1063/1.2000257.

[38] Jeremy O. Richardson. Full- and reduced-dimensionality instanton calculations of the tunnelling splitting in the formic acid dimer. Phys. Chem. Chem. Phys., 19:966-970, 2017. doi: 10.1039/C6CP07808G. URL http: //dx.doi.org/10.1039/C6CP07808G.

[39] Zorka Smedarchina, Antonio Fernandez-Ramos, and Willem Siebrand. Calculation of the tunneling splitting in the zero-point level and co-stretch fundamental of the formic acid dimer. Chemical Physics Letters, 395(4):339 - 345, 2004. ISSN 0009-2614. doi: https://doi.org/10.1016/j.cplett.2004.07.080. URL http://www.sciencedirect. com/science/article/pii/S0009261404011406.

[40] I.V. Kochikov, Yu.I. Tarasov, N. Vogt, and V.P. Spiridonov. Large-amplitude motion in 1,4-cyclohexadiene and 1,4-dioxin: theoretical background for joint treatment of spectroscopic, electron diffraction and ab initio data. Journal of Molecular Structure, 607(2):163 - 174, 2002. ISSN 0022-2860. doi: https://doi.org/10.1016/ S0022-2860(01)00914-0. URL http://www. sciencedirect.com/science/article/pii/S0022286001009140.

[41] Igor V. Kochikov and Yurii I. Tarasov. Equilibrium structure and internal rotation in b2f4 from electron diffraction and spectroscopic data and quantum chemical calculations. Structural Chemistry, 14(2):227-238, Apr 2003. ISSN 1572-9001. doi: 10.1023/A:1022102918764. URL https ://doi.org/10.1023/A:1022102918764.

[42] Frank Neese. The orca program system. Wiley Interdisciplinary Reviews: Computational Molecular Science, 2 (1):73-78, . doi: 10.1002/wcms.81. URL https://onlinelibrary.wiley.com/doi/abs/10.1002/wcms.81.

[43] John P. Perdew, Kieron Burke, and Matthias Ernzerhof. Generalized gradient approximation made simple [phys. rev. lett. 77, 3865 (1996)]. Phys. Rev. Lett., 78:1396-1396, Feb 1997. doi: 10.1103/PhysRevLett.78.1396. URL https://link.aps.org/doi/10.1103/PhysRevLett.78.1396

[44] Carlo Adamo and Vincenzo Barone. Toward reliable density functional methods without adjustable parameters: The pbe0 model. The Journal of Chemical Physics, 110(13):6158-6170, 1999. doi: 10.1063/1.478522. URL http://scitation.aip.org/content/aip/journal/jcp/110/13/10.1063/1.478522

[45] A. D. Becke. Density-functional exchange-energy approximation with correct asymptotic behavior. Phys. Rev. A, 38:3098-3100, Sep 1988. doi: 10.1103/PhysRevA.38.3098. URL https://link.aps.org/doi/10.1103/ PhysRevA.38.3098.

[46] Chengteh Lee, Weitao Yang, and Robert G. Parr. Development of the colle-salvetti correlation-energy formula into a functional of the electron density. Phys. Rev. B, 37:785-789, Jan 1988. doi: 10.1103/physrevb.37.785. URL http://link.aps.org/doi/10.1103/PhysRevB.37.785

[47] Axel D. Becke. Density-functional thermochemistry. iii. the role of exact exchange. The Journal of Chemical Physics, 98(7):5648-5652, 1993. doi: 10.1063/1.464913. URL http://link.aip.org/link/?JCP/98/5648/1.

[48] Takeshi Yanai, David P Tew, and Nicholas C Handy. A new hybrid exchange-correlation functional using the coulomb-attenuating method (cam-b3lyp). Chemical Physics Letters, 393(1):51 - 57, 2004. ISSN 0009-2614. doi: https://doi.org/10.1016/j.cplett.2004.06.011. URL http://www.sciencedirect.com/science/article/ pii/S0009261404008620.

[49] Stefan Grimme. Semiempirical hybrid density functional with perturbative second-order correlation. The Journal of Chemical Physics, 124(3):034108, 2006. doi: 10.1063/1.2148954. URL https://doi.org/10.1063/1.2148954.

[50] Tobias Schwabe and Stefan Grimme. Towards chemical accuracy for the thermodynamics of large molecules: new hybrid density functionals including non-local correlation effects. Phys. Chem. Chem. Phys., 8:4398-4401, 2006. doi: 10.1039/B608478H. URL http://dx.doi.org/10.1039/B608478H

[51] Chr. Møller and M. S. Plesset. Note on an approximation treatment for many-electron systems. Physical Review, 46(7):618-622, Oct 1934. doi: 10.1103/physrev.46.618.

[52] Stefan Grimme. Improved second-order møller-plesset perturbation theory by separate scaling of parallel- and antiparallel-spin pair correlation energies. The Journal of Chemical Physics, 118(20):9095-9102, 2003. doi: 10.1063/1.1569242. URL https://doi.org/10.1063/1.1569242. 
[53] Rodney J. Bartlett and Monika Musiał. Coupled-cluster theory in quantum chemistry. Rev. Mod. Phys., 79:291352, Feb 2007. doi: 10.1103/RevModPhys.79.291. URL https://link.aps.org/doi/10.1103/RevModPhys.79. 291.

[54] Stefan Grimme, Stephan Ehrlich, and Lars Goerigk. Effect of the damping function in dispersion corrected density functional theory. Journal of Computational Chemistry, 32(7):1456-1465, 2011. doi: 10.1002/jcc.21759. URL https://onlinelibrary.wiley.com/doi/abs/10.1002/jcc.21759

[55] J. L. Whitten. Coulombic potential energy integrals and approximations. The Journal of Chemical Physics, 58 (10):4496-4501, 1973. doi: 10.1063/1.1679012. URL https://doi.org/10.1063/1.1679012

[56] B. I. Dunlap, J. W. D. Connolly, and J. R. Sabin. On some approximations in applications of x $\alpha$ theory. The Journal of Chemical Physics, 71(8):3396-3402, 1979. doi: 10.1063/1.438728. URL https://doi.org/10.1063/ 1.438728

[57] Frank Neese. An improvement of the resolution of the identity approximation for the formation of the coulomb matrix. Journal of Computational Chemistry, 24(14):1740-1747, . doi: 10.1002/jcc.10318. URL https:// onlinelibrary.wiley.com/doi/abs/10.1002/jcc.10318.

[58] Frank Neese, Frank Wennmohs, Andreas Hansen, and Ute Becker. Efficient, approximate and parallel hartreefock and hybrid dft calculations. a 'chain-of-spheres' algorithm for the hartree-fock exchange. Chemical Physics, 356(1):98 - 109, 2009. ISSN 0301-0104. doi: https://doi.org/10.1016/j.chemphys.2008.10.036. URL http://www . sciencedirect.com/science/article/pii/S0301010408005089. Moving Frontiers in Quantum Chemistry:.

[59] Simone Kossmann and Frank Neese. Comparison of two efficient approximate hartee-fock approaches. Chemical Physics Letters, 481(4):240 - 243, 2009. ISSN 0009-2614. doi: https://doi.org/10.1016/j.cplett.2009.09.073. URL http://www.sciencedirect.com/science/article/pii/S0009261409011798.

[60] Simone Kossmann and Frank Neese. Efficient structure optimization with second-order many-body perturbation theory: The rijcosx-mp2 method. Journal of Chemical Theory and Computation, 6(8):2325-2338, 2010. doi: 10.1021/ct100199k. URL https://doi.org/10.1021/ct100199k. PMID: 26613489.

[61] Róbert Izsák and Frank Neese. An overlap fitted chain of spheres exchange method. The Journal of Chemical Physics, 135(14):144105, 2011. doi: 10.1063/1.3646921. URL https://doi.org/10.1063/1.3646921.

[62] Florian Weigend and Reinhart Ahlrichs. Balanced basis sets of split valence, triple zeta valence and quadruple zeta valence quality for $\mathrm{h}$ to rn: Design and assessment of accuracy. Phys. Chem. Chem. Phys., 7:3297-3305, 2005. doi: 10.1039/B508541A. URL http://dx.doi.org/10.1039/B508541A.

[63] Florian Weigend. Accurate coulomb-fitting basis sets for h to rn. Phys. Chem. Chem. Phys., 8:1057-1065, 2006. doi: 10.1039/B515623H. URL http://dx.doi.org/10.1039/B515623H

[64] Arnim Hellweg, Christof Hättig, Sebastian Höfener, and Wim Klopper. Optimized accurate auxiliary basis sets for ri-mp2 and ri-cc2 calculations for the atoms rb to rn. Theoretical Chemistry Accounts, 117(4):587-597, Apr 2007. ISSN 1432-2234. doi: 10.1007/s00214-007-0250-5. URL https://doi.org/10.1007/s00214-007-0250-5.

[65] Florian Weigend. Hartree-fock exchange fitting basis sets for h to rn $\dagger$. Journal of Computational Chemistry, 29 (2):167-175, 2008. doi: 10.1002/jcc.20702. URL https://onlinelibrary.wiley.com/doi/abs/10.1002/jcc. 20702 .

[66] William H. Miller, Nicholas C. Handy, and John E. Adams. Reaction path hamiltonian for polyatomic molecules. The Journal of Chemical Physics, 72(1):99-112, 1980. doi: 10.1063/1.438959. URL https://doi.org/10.1063/ 1.438959 .

[67] Tucker Carrington and William H. Miller. Reaction surface hamiltonian for the dynamics of reactions in polyatomic systems. The Journal of Chemical Physics, 81(9):3942-3950, 1984. doi: 10.1063/1.448187. URL https://doi.org/10.1063/1.448187.

[68] Norihiro Shida, Paul F. Barbara, and Jan Almlöf. A reaction surface hamiltonian treatment of the double proton transfer of formic acid dimer. The Journal of Chemical Physics, 94(5):3633-3643, 1991. doi: 10.1063/1.459734. URL https ://doi .org/10.1063/1.459734.

[69] Tucker Carrington and William H. Miller. Reaction surface description of intramolecular hydrogen atom transfer in malonaldehyde. The Journal of Chemical Physics, 84(8):4364-4370, 1986. doi: 10.1063/1.450058. URL https://doi.org/10.1063/1.450058

[70] R. Meyer and Hs. H. Günthard. General internal motion of molecules, classical and quantum-mechanical hamiltonian. The Journal of Chemical Physics, 49(4):1510-1520, 1968. doi: 10.1063/1.1670272. URL https://doi.org/10.1063/1.1670272 
[71] E.B. Wilson, J.C. Decius, and P.C. Cross. Molecular Vibrations: The Theory of Infrared and Raman Vibrational Spectra. Dover Books on Chemistry Series. Dover Publications, 1980. ISBN 9780486639413. URL https: //books.google.de/books?id=CPkvsDrPiv0C.

[72] Joseph W. Ochterski. Vibrational analysis in gaussian, 1999. URL https://gaussian.com/vib/.

[73] Denis S. Tikhonov. https://stash.desy.de/projects/MOLINC, 2020.

[74] Daniel T. Colbert and William H. Miller. A novel discrete variable representation for quantum mechanical reactive scattering via the s-matrix kohn method. The Journal of Chemical Physics, 96(3):1982-1991, 1992. doi: 10.1063/1.462100. URL https://doi.org/10.1063/1.462100.

[75] John P. Perdew and Karla Schmidt. Jacob's ladder of density functional approximations for the exchangecorrelation energy. AIP Conference Proceedings, 577(1):1-20, 2001. doi: 10.1063/1.1390175. URL https://aip. scitation.org/doi/abs/10.1063/1.1390175.

[76] Peter Kraus. Basis set extrapolations for density functional theory. Journal of Chemical Theory and Computation, 16(9):5712-5722, 2020. doi: 10.1021/acs.jctc.0c00684. URL https://doi.org/10.1021/acs.jctc.0c00684. PMID: 32790303.

[77] Sebastian Dohm, Andreas Hansen, Marc Steinmetz, Stefan Grimme, and Marek P. Checinski. Comprehensive thermochemical benchmark set of realistic closed-shell metal organic reactions. Journal of Chemical Theory and Computation, 14(5):2596-2608, 2018. doi: 10.1021/acs.jctc.7b01183. URL https://doi.org/10.1021/acs . jctc.7b01183. PMID: 29565586. 